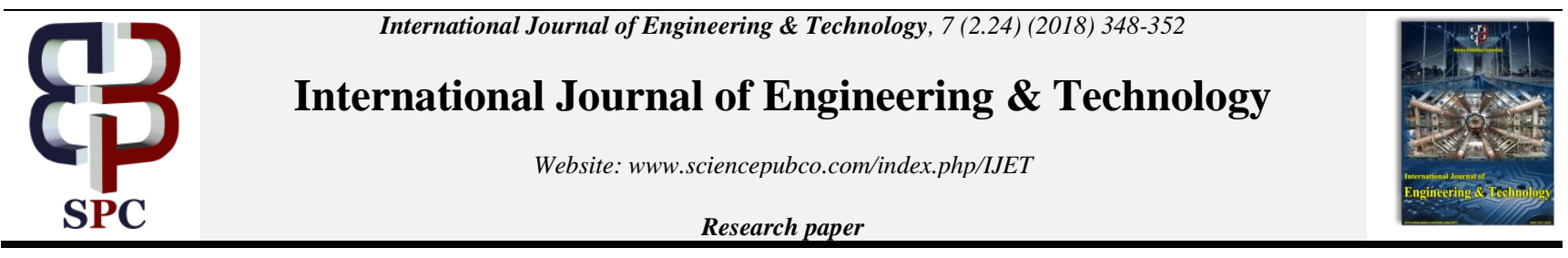

\title{
Three Level Optimization Models of Scaled Gabor Features for Facial Expression Recognition
}

\author{
Neha $^{1 *}$, Pratistha Mathur ${ }^{2}$ \\ ${ }^{1}$ Research Scholar Banasthali Vidyapith, Rajasthan, India \\ ${ }^{2}$ Associate Professor SKITJaipur \\ *Email: neha123jaipur@gmail.com
}

\begin{abstract}
The area of computer vision and machine learning for pattern recognition has witnessed the need for research for the development of algorithms for different applications such as human-computer interaction, automated access control and surveillance. In the field of computer vision Facial Expression Recognition has attracted the researcher's interest. This paper presents a novel feature extraction technique: Gabor-Average-DWT-DCT for automatic facial expression recognition from a person's face image invariant of illumination. Facial Emotions have different edge and texture pattern. Gabor filter is able to extract edges and texture pattern of faces but with problem of huge dimension and high redundancy. The problem of huge dimension and high redundancy is reduced by proposed Average-DWT-DCT feature reduction technique in order to increase accuracy of system. Proposed Gabor- Average -DWT-DCT provides a compact feature vector for reducing response time of system compared to existing Gabor based expression classification. Detailed quantitative analysis is done and results that the average recognition rate of proposed technique is better than state of art results.
\end{abstract}

Keywords: Facial Emotion Recognition, Gabor Filter, DWT, DCT.

\section{Introduction}

Every object, event and process has some pattern to which we can assign a name like finger prints, words (hand written), human faces, speech signals, DNA sequences etc. Pattern Recognition is a process through which patterns in objects and data are found. This is a study of observation of the environment by the machine, learning from overlap and mix pattern with background and evaluate the threshold for making decision of categories from unseen of data. Pattern recognition is a process which enables classification of an object in one of the category using characteristics or features extracted from the object. There are wide applications of pattern recognition as Face Biometric Identification, Facial Gesture Recognition and Ear Biometric Recognition. Today many institutions /organizations are using biometric for maintaining employee present and other activities like stock market prediction, loan assessment and signature recognition are also done using pattern recognition, even when we go to see the doctor our health is also measured according to the patterns like weight, height etc. Among all Facial expression recognition system is an application which is used for designing of computer based human computer interface, machine learning and artificial system.

Human faces have various patterns or encoded information as gender, unique identity, gesture and age. Retrieval of this information from face image has wide applications such as human machine interactions, behavioral analysis, biometric authentication and de identification. Facial Emotion Recognition is key vital research topic for in current scenario and has wide application as activity recognition, human-computer interaction. Various re- search techniques are proposed for facial expression recognition but no method is able to provide high level of accuracy. The methodology of facial expression recognition includes facial expression image acquisition, preprocessing, feature extraction and classification. The facial expression of individual is changed slightly over the years so it is problem for identification after a long time of span while iris and ear are not changed over a long time [1]. Facial Expression Recognition is used in machine learning application as robo-human communication and machine based interactions. Research Issues in Facial Expression Recognition is enhancing the accuracy which depends on preprocessing, feature extraction and classification. Among these Feature Extraction is the key stage. Optimization of Feature Extraction is a vital process which can change the accuracy significantly. The different features of unique gesture determination are edges pattern and skin color variation in which edge pattern is a key features. Yamada [2] suggested that color information of gesture face is key information for the classification of expression. Fengjun Chen et al. [3] used 14 wavelet decomposed regions for enhance the accuracy of facial gesture recognition. Sameer S. Kulkarni and John moriarty [4] analyzed impact of image block size using discrete cosine transform based feature extraction for facial expression recognition for determination of best block size to achieve best accuracy. Bin Jiang et al. [5] conclude that DCT based feature extraction technique have more accuracy compared to 2D Principle component analysis feature extraction technique for facial expression recognition. Xiaoli Li et al. [6] has identified the high dimension and high redundancy of Gabor filter feature extraction technique and concludes that local Gabor filters is more beneficial for less running time of feature extracting process. Behnam Kabirian Dehkordi et al. [7] presented a Gabor filter technique for geometric part as such as nose, ear, mouth and eyebrows (with eye) of facial expres- 
sion rather than whole space of face. LinLin Shen and Li Bai [8] have proposed combined Gabor wavelets and Kernel methods in order to achieve highly discriminative features for face recognition and added that Gabor is more robust for illumination changes compared to DCT and DWT based techniques. Hybrid based feature extraction [9] is used to extract features based on Gabor with DWT, DCT and Gaussian for gesture recognition. The proposed paper analyzed problem of Gabor filter feature extraction technique and optimized the features extraction stage through Gabor features for improving accuracy of gesture recognition.

Main Contribution: In our paper, we analyzed problem of huge dimension and high redundancy in Gabor filter feature extraction technique and optimized the features by proposed Average-DWTDW-DCT technique in order to improve accuracy and run time of system effectively.

\section{Feature Extraction Techniques}

As we have studied that Feature Extraction is the main stage of emotion recognition on which accuracy is depended and by improving the feature extraction techniques, we can improve the accuracy of emotion recognition system. Recently, there have been various methods of feature extraction.

Here we are discussing related transform based feature extraction and Gabor feature extraction techniques which are used in proposed feature optimization technique.

\subsection{Gabor Filter Feature Extraction Techniques}

Gabor filter is used generally for analysis of texture, detection of edges, extraction of features, estimation of disparity (in stereo vision), etc. Whenever an image is convolved with Gabor kernel, it generates highest response at edges and at points where texture changes.

Gabor kernel is represented by following equation 1

$\Psi(\mathrm{x}, \mathrm{y}, \lambda, \theta)=\frac{1}{2 \pi s_{-} x s_{-} y} e^{-\frac{1}{2}}\left(\frac{x_{-} 1^{\wedge} 2}{s_{-} x^{\wedge} 2}+\frac{y_{-} 1^{\wedge} 2}{s_{-} y^{\wedge} 2}\right) e^{j \frac{2 \pi x \_1}{\lambda}}$

where $\lambda=1 / \mathrm{f}$ and $\mathrm{f}$ denotes the frequency of sinusoidal wave, $\theta$ represented the projection angle of Gabor kernel and $S_{\mathrm{x}}, \mathrm{S}_{\mathrm{y}}$ are the standard deviations in 2D-gaussion distribution for $\mathrm{x}$ and $\mathrm{y}$ directions respectively. The parameters $x^{\prime}$ and $y^{\prime}$ are evaluated from $\mathrm{x}, \mathrm{y}$ and angle as equation 2 [10].

$$
\mathrm{x}^{\prime}=\mathrm{x} \cos \theta+\mathrm{y} \sin \theta \quad \mathrm{y}^{\prime}=-\mathrm{x} \cos \theta+\mathrm{y} \sin \theta
$$

The Gabor features are calculated by convolution of input image with Gabor filter bank [11] which generates horizontal and vertical edges and texture pattern. $\mathrm{I}(\mathrm{x}, \mathrm{y})$ is denoted grey-scale face image of size $a^{*} b$ pixels in spatial domain. Gabor kernel $\Psi(x, y)$ have complex number so the convolution operation is performed separately for real and imaginary part which is shown in equation 3 and 4 respectively. Finally the amplitudes of Gabor confidents are shown in equations 5. Finally the amplitudes of Gabor confidents are shown in equations 6 [10].

$$
\begin{aligned}
& \mathrm{G}_{\mathrm{u}, \mathrm{v}}(\mathrm{x}, \mathrm{y})=\Psi(\mathrm{x}, \mathrm{y}) * \mathrm{I}(\mathrm{x}, \mathrm{y}) \\
& \operatorname{Re}(\mathrm{O}(\mathrm{x}, \mathrm{y}))_{\mathrm{m}, \mathrm{n}}=\mathrm{I}(\mathrm{x}, \mathrm{y}) * \operatorname{Re}\left(\psi\left(\mathrm{x}, \mathrm{y}, \lambda_{\mathrm{m}}, \theta_{\mathrm{n}}\right)\right) \\
& \operatorname{Im}(\mathrm{O}(\mathrm{x}, \mathrm{y}))_{\mathrm{m}, \mathrm{n}}=\mathrm{I}(\mathrm{x}, \mathrm{y}) * \operatorname{Im}\left(\psi\left(\mathrm{x}, \mathrm{y}, \lambda_{\mathrm{m}}, \theta_{\mathrm{n}}\right)\right) \\
& |\mathrm{O}(\mathrm{x}, \mathrm{y})|_{\mathrm{m}, \mathrm{n}}=\left(\left(\operatorname{Re}(\mathrm{O}(\mathrm{x}, \mathrm{y}))_{\mathrm{m}, \mathrm{n}}\right)^{2}+\left(\operatorname{Im}(\mathrm{O}(\mathrm{x}, \mathrm{y}))_{\mathrm{m}, \mathrm{n}}\right)^{2}\right)^{1 / 2}
\end{aligned}
$$

\subsection{Facial Expression Recognition with Discrete Wavelet} Transform
In today's world most of the people use images to attract others. Mostly data or images are not used in their original form they are compressed and then sent or shared. Wavelet transform is defined as decomposition of image into low frequency coefficients and high frequency coefficients. The image is regenerated from the low and high frequency coefficients and process is called as the inverse discrete wavelet transforms [13]. DWT breaks a digital signal into sub bands. In this way sub bands are able to have better frequency resolutions. DWT is a technique that is used to transform the pixels of an image to the wavelets [14].

DWT transformation converts the facial gesture image into four different frequency sub bands as $\mathrm{LL}, \mathrm{LH}, \mathrm{HL}$ and $\mathrm{HH}$ as given in figure 1 .

\begin{tabular}{|c|c|}
\hline A Sub band & H Sub band \\
\hline V Sub band & D Sub band \\
\hline
\end{tabular}

Fig. 1: frequency Sub band of DWT

\subsection{Discrete Cosine Transform based feature extraction technique}

As DWT transformation, DCT is also used transform an image from space domain to frequency domain. Discrete Cosine Transform allows increased throughput through transmission medium, video and audio compression, through this medium it makes multimedia systems very efficient. DCT extract the energy information of the image and low frequency features is arranged in upper left Corner of the DCT matrix and high frequency features is arranged into right bottom of DCT matrix [16, 17]. Discrete cosine transform is defined [18] as equation (7).

$\mathrm{F}(\mathrm{i}, \mathrm{j})=\alpha(\mathrm{i}) \alpha(\mathrm{j}) \Sigma \quad \sum_{x=0}^{A-1} \sum_{y=0}^{B-1} f(x, y) \cos \left[\frac{\pi(2 \mathrm{x}+1) i}{2 A}\right] \cos \left[\frac{\pi(2 y+1) j}{2 B}\right]$

Where $i=0,1,2 \ldots$ A- 1 and $j=0,1,2 \ldots B-1$.

$\alpha(\mathrm{i})$ and $\alpha(\mathrm{j})=\left\{\begin{array}{l}\sqrt{\frac{1}{A}}, \text { for } i, j=0 \\ \sqrt{\frac{2}{A}}, \text { for } i, j \neq 0\end{array}\right.$

\subsection{Feature Reduction Techniques}

If the dimension of features extracted in feature extraction step is high the performance of system will be affected so Feature Reduction techniques are used to reduce the dimension of extracted features. Different techniques used for dimension reduction are principle component analysis, linear discernment analysis, Generalized discriminate analysis, canonical correlation analysis, independent component analysis.

Principle Component Analysis: It is a techniques based on statistical too and correlations among features. PCA is used for dimensionality reduction of data by eliminating non-essential information. On the each output matrix of Gabor Mean feature optimization process Gmean, Principle component analysis is applied, which generate eigen vectors with corresponding different Eigen values.

The covariance matrix [19] $\operatorname{cov}(\mathrm{x}, \mathrm{y})$ is generated using Gmean $^{\mathrm{T} *}$ Gmean. On applying PCA on nxp size of feature matrix, it generate Gabor Filter mean PCA Feature Extraction for Gender Recognition $\mathrm{p}^{*} \mathrm{p}$ size PCA coefficient matrix in which each col- 
umn represents eigen vector corresponding to decreasing variance. From these eigen vectors, eigen value corresponding to significant eigen values are selected which is our reduced features for classification. The significance level of PCA is calculated using equation 8 .

$\varepsilon=\frac{\sum_{i}^{m} \wedge_{i}}{\sum_{j}^{n} \wedge_{j}}$

Where $\mathrm{m}<=\mathrm{n}$ and $0<\varepsilon<1$ where $\mathrm{i}$ and $\mathrm{j}$ represents the Eigen value of $i^{\text {th }}$ and $j^{\text {th }}$ order in sort of amplitude and $m<=n$. Eigen vector corresponding to high amplitude of Eigen values gives higher variance information in image correlated features. So these are selected and remaining is discarded in order to reduce the dimension.

\section{Proposed Work}

There are various methods used for feature extraction. Among all these Gabor features are broadly used as the features of facial expressions. Gabor Filter is more robust against illumination changes compared to other techniques. It has good characteristics of selecting information from spatial position and different orientation. In the Gabor filter feature extraction, edge information is generated using Gabor projection on image but problem of increasing of redundancy can be responsible for reducing the recognition rate. Gabor has a major problem issue of high dimension and high redundancy while it provides high energy difference of shape information and non shape information. Redundancy and Dimension must be decreased using some optimization techniques. In general a single level optimization of feature selection technique is used. In this research we have proposed new model which uses multiple level of feature optimization for facial expression analysis application. Hence the proposed methodology is as follows:

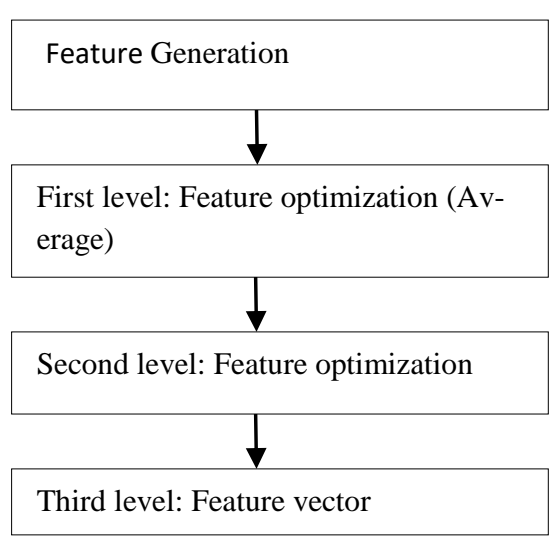

Fig. 2: Proposed three tier model of feature extraction and optimization

In the proposed methodology of feature optimization, Gabor equation is projected 7 times on 3 different scaled image using 7 different orientations which provide $7 * 3=21$ Gabor feature matrices. These Gabor feature matrices coves 4 dimension space with huge redundant features which are optimized by following proposed 3 level feature optimization techniques in order to achieve compact size of final feature and high level unique pattern of gesture. The proposed model is shown in figure 3.

$1^{\text {st }}$ Level: Average filtering: Gabor feature are optimized using average feature extraction process in which corresponding average of respective Gabor wavelet features are calculated along orientation. Output of this stage is denoted as Gavg which is a collection of matrices. $2^{\text {nd }}$ Level: DWT filtering: On the output of second stage feature optimization process, discrete wavelet transform is applied, which transform it into 4 frequency band as LL, LH, HL and HH sub band. LL band have low frequency sub band (Approximation coefficients) which shows edge pattern of gesture while other bands shows detail information in image. From Low frequency region, LL sub and is extracted and passed to next optimization process.

$3^{\text {rd }}$ Level: DCT filtering: On each extracted LL matrix of wavelet transform matrix, discrete cosine transform is applied which transform average Gabor wavelet matrix into cosine frequency coefficient matrix. Concept says that shape information of features is represented by low frequency region and Low frequency region is extracted from top left position as zigzag selection approach from average Gabor wavelet cosine matrix.

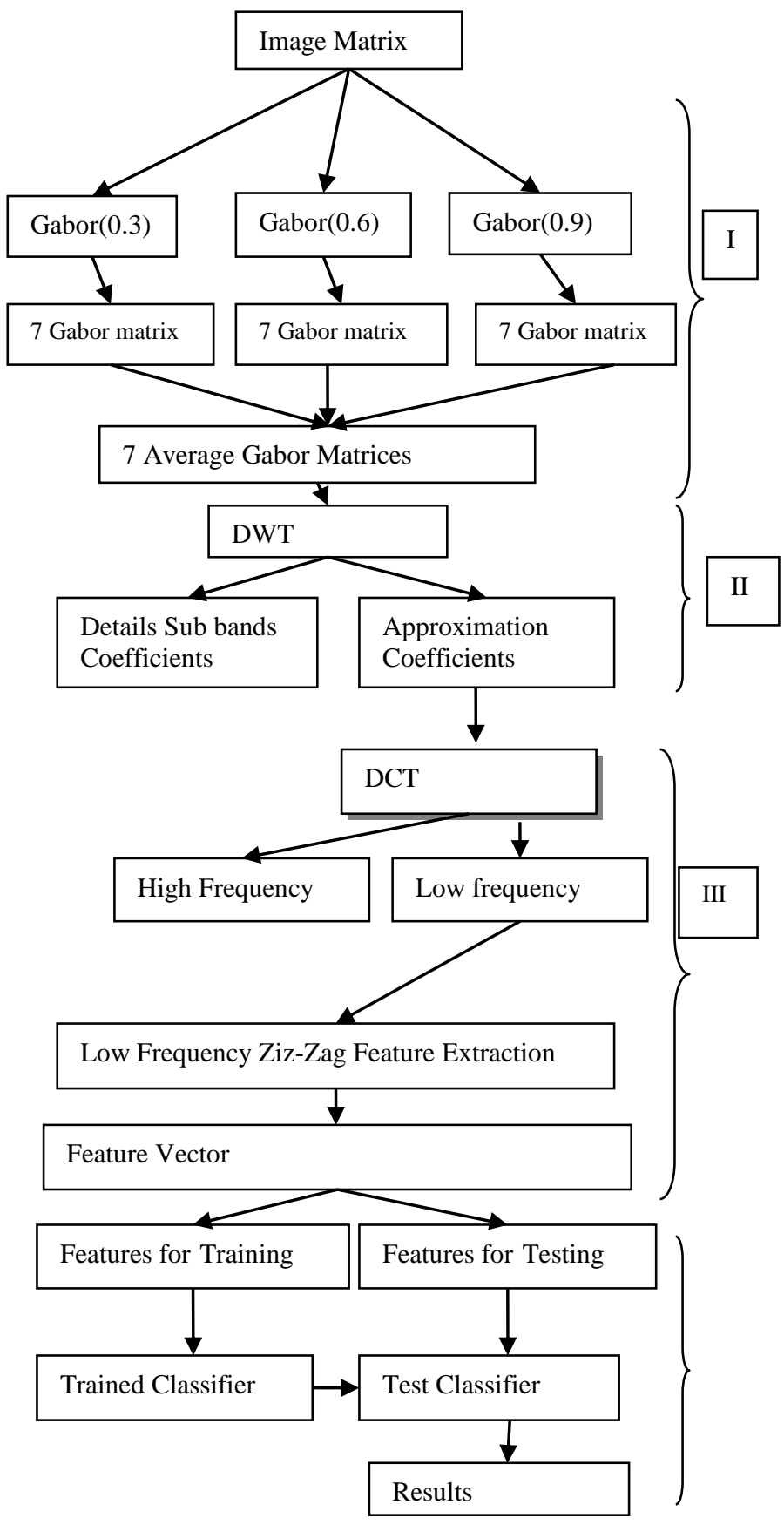

Fig. 3: Proposed model of facial gesture recognition 


\section{Algorithm of Proposed Gabor Approach}

Step 1: Input: Image Img is read.

Step2: Image is converted into gray scale.

Step 3: Set $\mathrm{m}($ scale $)=[0.3,0.6,0.9]$;

Repeat for $i=1$ to $m$

Img $_{\text {resize }}=$ resized the image with scale $(i)$

Set $n($ theta $)=[0,30,60,90,120,150,180]$

Repeat for $j=1$ to $n$

Gabor filtering is applied on each rescaled image with theta end loop

$1^{\text {st }}$ Level Optimization: corresponding average of respective Gabor features are calculated along orientation. In this process, dimension and redundancy of Gabor matrices is reduced by number of angles applied in feature generation and selection process

$2^{\text {nd }}$ Level Optimization: Apply DWT transformation on average Gabor feature matrix iteratively and Low frequency coefficients matrix (LL) are extracted.

$3^{\text {rd }}$ Level Optimization: Apply DCT transformation of extracted $L L$ $d w t$ coefficient matrix and low frequency prominent features are selected using zigzag manner. Finally features are merged into feature vector.

End loop.

\section{Experiment results \& analysis}

Proposed technique is implemented in MATLAB and JAFEE dataset is used with adaboost classifier with $80 / 20$ training/testing ratio. A result of proposed technique is shown in Table 2 . In the JAFFE and CK dataset, face is located at canter position with same size so it can be cropped using static pixel bounding box method. If face is not located at fixed position in facial image and size is also varied then face detection is necessary to locate the face location. Viola Jones method is widely used face detection. Facial gesture image I is taken input of sized 256x256 pixels (for example it is supposed we applied proposed method on JAFFE dataset), if the images are color images then stored in 3D Array and it is first converted into gray scale. JAFFE dataset have gray scale images and store in $2 \mathrm{D}$ matrix so no need to convert into gray scale. The proposed scale Gabor-DWT-DCT feature extraction method affects the size, dimension and redundancy of features as following:

Preprocessing is applied to remove background part of gesture image and convert into gray scale. In our experiment images are cropped and after cropping image is resized 200*200 after that Gabor features are generated with different scaled image which generate three different sized images which are described in Table 1 .

Input image of size $200 \times 200$ generate $(60 \times 60+120 \times 120+180 \times 180)$ x7 Gabor features after Gabor projection and after that Proposed average (mean) feature extraction is applied for reducing dimensions. After corresponding average of respective Gabor features we get average matrix of size $60 \times 60,120 \times 120$, and $180 \times 180$ coefficients. Total feature coefficients at this stage are $60 * 60+120 * 120+180 * 180==50400$

2D-Discreate Wavelet Transform (DWT) is applied on each average-Gabor feature matrix and most characteristics features of edge and texture are extracted from low frequency features (LL sub band). After extraction, dimension of feature is reduced by $1 / 4$ so we have feature matrix of $30 \times 30,60 * 60,90 * 90$.

Total feature coefficients till above step $=30 \times 30+60 * 60+$ $90 * 90=12600$

2D-Discreate cosine transform (DCT) is applied on each extracted LL block and most characteristics features of edge and texture are extracted in zigzag order and put into feature vector. We have extracted total 512 low features from DCT frequency matrix using zigzag feature selection approach. After proposed three levels optimization process converts feature size of 512 which is compact and concise. This feature vector with class level is passed to adaboost classifier for training. After training Feature vector of test images is passed to classifier with train weights to determine gesture among 7 different possible classes.

Table 1: Sizes of images at Scale $=0.3,0.6$ and 0.9

\begin{tabular}{ll}
\hline Environmental Parameters & Value \\
\hline Sizes of the images & JAFEE Dataset \\
No. of Images & 213 \\
No. of Classes & 7 \\
Original image(Size) & $256 \times 256$ \\
After pre-processing(Size) & $200 \times 200$ \\
At Scale 0.3(Size) & $60 \times 60$ \\
At Scale 0.6(size) & $120 \times 120$ \\
At Scale 0.9(Size) & $180 \times 180$ \\
\hline
\end{tabular}

Table 2: Performance comparison techniques

\begin{tabular}{lc}
\hline \hline \multicolumn{1}{c}{ Methods } & $\begin{array}{c}\text { Avg. } \\
\text { Recognition } \\
\text { Rate }(\%)\end{array}$ \\
\hline Gabor + Average DCT & 83.5 \\
DCT feature extraction based facial expres- & 58.3 \\
sion recognition & \\
Gabor filter + PCA based facial expression & 80 \\
recognition & 70 \\
Log Gabor + PCA & 90.55 \\
Gabor amplitude + PCA & 92.37 \\
Gabor phase + PCA & 88.4 \\
LBP + SVM & 88.25 \\
WPCA & 91.9 \\
Geometric Gabor + PZMI & 80 \\
DCT Zigzag Feature Extraction Technique & 92.85 \\
Proposed Gabor + Average + DWT+ DCT & \\
\hline
\end{tabular}

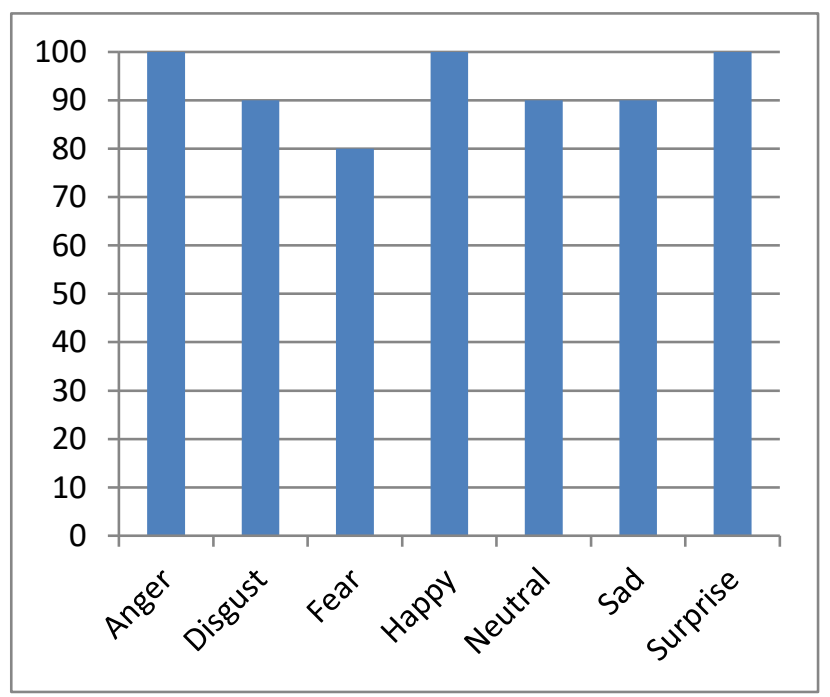

Fig. 4: Correct accuracy for each expression of proposed technique 


\section{Conclusion}

From the results achieved by different technique, it is concluding that Gabor feature extractions have higher accuracy compared to discrete wavelet transform and discrete cosine transform. The analysis of better result of Gabor features, it is analyzed that Gabor is better extraction technique for edge or shape features extraction compared to DWT and DCT feature extraction. In DWT and DCT feature extraction, feature are extracted from Low frequency feature sub band (LL) and other frequency band are discarded so some of feature are lost which is cause of lower accuracy compare to Gabor. Gabor with scale projection achieved better accuracy compared to Gabor without scale. The problem with Gabor filter is generation of high redundant feature and huge dimension of feature matrix which is reduced effectively using proposed 3 level optimization using average, DWT and DCT filtering process. The result shows that proposed technique achieved $92.85 \%$ accuracy on JAFEE dataset in terms of recognition rate. From the results, it is conclude that Accuracy of proposed 3 tier Gabor Average + DWT + DCT feature extraction technique achieved better accuracy compared to Gabor PCA and other feature extraction technique for facial expression recognition mentioned in table 2 . From figure 4 , it is analyzed that images of fear have minimum accuracy while anger, happy and surprise have maximum accuracy. Average of accuracy for all gestures is same as proposed recognition rate $92.85 \%$.

\section{References}

[1] Happy, S. L.and Aurobinda Routray. "Automatic facial expression recognition using features of salient facial patches." IEEE transactions on Affective Computing 6, no. 1 (2015): 1-12.

[2] Yamada, Takashi, and Tomio Watanabe. "Effects of facial color on virtual facial image synthesis for dynamic facial color and expression under laughing emotion." In Robot and Human Interactive Communication. ROMAN 2004. 13th IEEE International Workshop on, pp. 341-346. IEEE, 2004.

[3] Fengjun Chen, Zhiliang Wang, Zhengguang Xu, Donglin Wang, "Research on a Method of Facial Expression Recognition ", in IEEE The Ninth International Conference on Electronic Measurement \& Instruments, pp 225-230, IEEE, 2009.

[4] Kulkani, Sameer S., John Moriarty, and Chih-Cheng Hung. "The impact of Image block size on face feature extraction using discrete cosine transform." In IEEE Proceedings of the SoutheastCon 2010 (SoutheastCon), IEEE, 2010

[5] Jiang, Bin, Guo-Sheng Yang, and Huan-Long Zhang. "Comparative study of dimension reduction and recognition algorithms of DCT and 2DPCA.", 2008 International Conference on Machine Learning and Cybernetics, Vol. 1. IEEE, 2008.

[6] Xiaoli Li, Qiuqi Ruan and Chengxiong Ruan,"Facial Expression Recognition with Local Gabor Filters", Proceedings of 10th IEEE International Conference on Signal Processing (ICSP), pp. 1013-1016, 2010

[7] Behnam Kabirian Dehkordi Javad Haddadnia "Facial Expression Recognition With Optimum Accuracy Based on Gabor Filters and Geometric Features "IEEE Trans2nd International Conference on Signal Processing Systems (ICSPS), pp VI-731733, IEEE, 2010.

[8] LinLinShen and Li Bai, "Gabor Feature Based Face Recognition Using Kernel Methods", Proceedings of the Sixth IEEE International Conference on Automatic Face and Gesture Recognition (FGR'04), IEEE, 2004.

[9] Gupta, Sandeep K., ShubhLakshmi Agrwal, Yogesh K. Meena, and Neeta Nain. "A hybrid method of feature extraction for facial expression recognition." In Signal-Image Technology and Internet-Based Systems (SITIS), 2011 Seventh International Conference on, pp. 422-425. IEEE, 2011.

[10] Jiying Wu, Gaoyun An, and QiuqiRuan, "Independent Gabor Analysis of Discriminant Features Fusion for Face Recognition" Signal Processing Letters, Vol. 16, No. 2, IEEE, February 2009. Valstar, Michel. "Automatic facial expression analysis." In Understanding Facial Expressions in Communication, pp. 143-172. Springer India, 2015.

[11] Shilpa Choudhary, Kamlesh Lakhwani, and Shubhlakshmi Agrwal. "An efficient hybrid technique of feature extraction for facial expression recognition using AdaBoost Classifier." International Journal of Engineering Research \& Technology, issue: 1, vol no. 8, 2012.

[12] Lemley, Joseph, Sami Abdul-Wahid, Dipayan Banik, and Razvan Andonie, "Comparison of Recent Machine Learning Techniques for Gender Recognition from Facial Images" (2016).Meihua Wang, Hong Jiang and Ying Li," Face Recognition based on DWT/DCT and SVM ", IEEE International Conference on Computer Application and System Modeling 2010

[13] Hazar Mliki, Nesrine Fourati, Souhail Smaoui, Mohamed Hammami, "Automatic Facial Expression Recognition System", IEEE, 2013.

[14] Gupta, Sandeep K., ShubhLakshmi Agrwal, Yogesh K. Meena, and Neeta Nain, "A hybrid method of feature extraction for facial expression recognition" In Signal-Image Technology and Internet-Based Systems (SITIS), 2011 Seventh International Conference on, pp. 422-425. IEEE, 2011.

[15] Tian, Yingli, Takeo Kanade, and Jeffrey F. Cohn, "Facial expression recognition." In Handbook of face recognition, pp. 487-519. Springer London, 2011

[16] Jyoti poonia, Parvati Bhurani, Rohit Kumar, Shubh Lakshmi Agrawal, "Performance Review of IRIS Recognition Systems", International Journal of Computer Systems (IJCS), 2(12), pp: 564-566, December 2015.

[17] Shuyang Wang, JinzhengSha, Yun Fu, "Hirarchical Facial Expression animation by motion capture data", IEEE, 2014.

[18] SS Kulkarni, J. moriarty, chihcheng hung, "The impact of image clock size on face feature extraction using discrete cosine transform" in international conference on southeast con, pp. 98 $101,2010$.

[19] Rivera, Adin Ramirez, Jorge Rojas Castillo, and Oksam Oksam Chae. "Local directional number pattern for face analysis: Face and expression recognition." IEEE transactions on image processing 22, no. 5 (2013): 1740-1752.

[20] Whitehill, Jacob, Zewelanji Serpell, Yi-Ching Lin, Aysha Foster, and Javier R. Movellan. "The faces of engagement: Automatic recognition of student engagementfrom facial expressions." IEEE Transactions on Affective Computing 5, no. 1 (2014): 86-98.

[21] Valstar, Michel F., Bihan Jiang, Marc Mehu, Maja Pantic, and Klaus Scherer, "The first facial expression recognition and analysis challenge." In Automatic Face \& Gesture Recognition and Workshops (FG 2011), 2011 IEEE International Conference on, pp. 921-926. IEEE, 2011.

[22] Niu, Zhiguo, and Xuehong Qiu. "Facial expression recognition based on weighted principal component analysis and support vector machines." In2010 3rd International Conference on Advanced Computer Theory and Engineering (ICACTE), vol. 3, pp. V3-174. IEEE, 2010

[23] Dehkordi, Behnam Kabirian, and Javad Haddadnia. "Facial expression recognition with optimum accuracy based on Gabor filters and geometric features." In Signal Processing Systems (ICSPS), 2010 2nd International Conference on, vol. 1, pp. V1731. IEEE, 2010.

[24] Neha, Sandeep K. Gupta, Pratishta Mathur, "Performance Analysis of Feature Extraction Techniques for Facial Expression Recognition", International Journal of Computer Applications, 2017.

[25] Neha Janu, Pratishta Mathur, Sandeep K. Gupta, Shubhlakshmi Agrwal, "Performance Analysis of Frequency Domain based Feature Extraction Techniques for Facial Expression Recognition", 7th IEEE International Conference on Cloud Computing, Data Science \& Engineering, IEEE, 2017. 